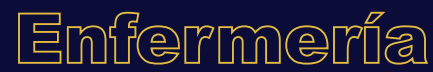

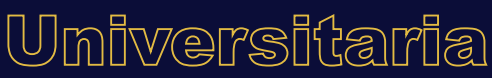

\section{Estudio fenomenológico: Soy padre y entré al parto, ifue un sueño hecho realidad!}

\author{
Phenomenological study: I am a father \\ and I observed the labor process. \\ It was a dream come true!
}

\section{Estudo fenomenológico: Sou pai e entrei em trabalho de parto, foi um sonho realizado!}

\section{C.E. Arévalo-Venegas ${ }^{a{ }^{1 *}}$, N. Castiblanco-López ${ }^{\mathrm{b}_{2}}$}

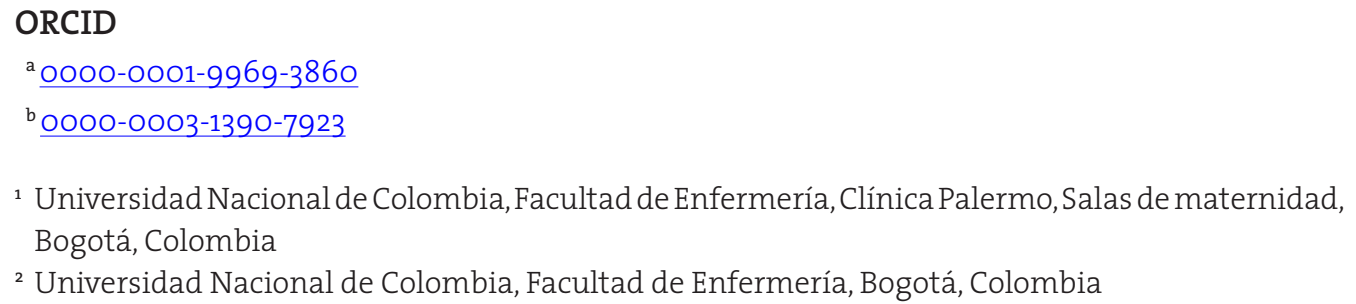
en instituciones colombianas aún son deficientes y carecen de soporte sólido en teorías de enfermería; sin embargo, a medida que avanza el mundo, los nuevos padres buscan involucrarse en el nacimiento de su hijo(a).

Objetivo: Comprender la experiencia de los padres que vivenciaron el nacimiento de su hijo(a) en una institución de salud en Bogotá, Colombia entre julio 2018 y marzo 2019. Método: Estudio cualitativo fenomenológico interpretativo que integró la Teoría de enfermería de desarrollo humano propuesto por Rosemarie Rizzo Parse. La información se

*Autora para correspondencia.Correo electrónico: cearevalov@unal.edu.co https://doi.org/10.22201/eneo.23958421e.2021.1.870

1665-7063/@ 2021 Universidad Nacional Autónoma de México, Escuela Nacional de Enfermería y Obstetricia. Este es un artículo Open Access bajo la licencia CC BY-NC-ND (http://creativecommons.org/licenses/by-nc-nd/4.o/). 
recolectó mediante entrevistas realizadas a ocho padres con observación no participante y participante, diarios de campo y llamadas telefónicas.

Resultados: La experiencia de los padres se consolidó en tres temas titulados: el parto de los padres, ahi estamos los tres y enfermería, un soporte excelente.

Discusión: Se observa una estructura nutrida de sentimientos, sensaciones, actitudes y valores de los padres, la cual es esencial para vivenciar la experiencia de la forma descrita. Conclusión: Los padres atravesaron el proceso de desarrollo humano, iniciaron por la estructuración del significado, es decir, el parto fue tan importante como su propia vida, pasando por la configuración de sus pautas rítmicas referidas a que eligieron estar presentes y, finalmente, co-trascendieron hacia la paternidad deseada. Los resultados guían el cuidado de enfermería de los padres que experimentan el nacimiento de sus hijos/as, basada en una presencia real. Además, evidencian la importancia de fortalecer políticas, planes y guías de enfermería.

Palabras clave: Acontecimientos que cambian la vida; paternidad; parto; teoría de enfermería; investigación cualitativa; Colombia.

\section{ABSTRACT}

Introduction: The implementation processes in the accompaniment of women in birth labor in Colombian institutions are deficient and without solid support in nursing theories, however, as the world progresses, new fathers seek to be involved in the birth of their child. Objective: To understand the experience of fathers who experienced the birth of their child in a health institution in Bogotá (Colombia) between July 2018 and March 2019.

Method: An interpretive phenomenological qualitative study that integrated the nursing theory of Human Development proposed by Rosemarie Rizzo Parse. The information was collected through interviews with 8 fathers with non-participant and participant observation, field diaries and phone calls.

Results: The father's experience was consolidated in 3 themes: the birth of the fathers, the three of us are there and nursing excellent support.

Discussion: The study allows us to penetrate into a nurtured structure of a father's feelings, sensations, attitudes, and values, essential to living the experience as described.

Conclusion: The parents who had the process of human development began with the structuring of the meaning it was as important as my own life, then they went through the configuration of their rhythmic guidelines that refers to they chose to be present and, finally, they co-transcended towards the desired parenthood. The results serve as a guide for nursing care of the parents who experience the birth of their children, based on a real presence. They also show the importance of strengthening nursing policies, plans, and guidelines.

Keywords: Life change events; paternity; parturition; nursing theory; qualitative research; Colombia.

RESUMO

Introdução: Os processos de implementação do acompanhamento de mulheres em trabalho de parto em instituições colombianas ainda são deficiente e carecem de sólido apoio nas 
teorias de enfermagem; no entanto, à medida que o mundo avança, novos pais procuram se envolver no nascimento de seus filhos.

Objetivo: Compreender a experiência de pais que vivenciaram o nascimento de um filho em uma instituição de saúde de Bogotá, Colômbia, entre julho de 2018 e março de 2019.

Método: Estudo fenomenológico interpretativo qualitativo que integrou a teoria do desenvolvimento humano em enfermagem proposta por Rosemarie Rizzo Parse. As informações foram coletadas por meio de entrevistas com 8 pais com observação não participante e participante, diários de campo e telefonemas.

Resultados: A experiência dos pais foi consolidada em 3 temas: entrega dos pais, nós três estamos lá e amamentando um excelente apoio.

Discussão: Uma estrutura nutrida dos sentimentos, sensações, atitudes e valores dos pais é permeada essencial para viver a experiência da maneira descrita.

Conclusão: Os pais passaram pelo processo de desenvolvimento humano, iniciando pela estruturação do significado era tão importante quanto a própria vida, passando pela configuração de suas orientações rítmicas que remete a escolheram estar presentes e, por fim, eles co-transcendido para a paternidade desejada. Os resultados norteiam o cuidado aos pais que vivenciam o nascimento de seus filhos, a partir de uma presença real. Também mostram a importância do fortalecimento das políticas, planos e diretrizes de enfermagem. Palavras chave: Acontecimentos que mudam a vida; paternidade; parto; teoria de enfermagem; pesquisa qualitativa; Colômbia.

\section{INTRODUCCIÓN}

El nacimiento de un hijo es un acontecimiento único y crucial en la vida de una pareja; pero se le ha dado escasa participación al hombre con argumentos poco sólidos'; a su vez, se ha dejado sola a la mujer durante el parto con consecuencias para la salud materno infantil2. Por lo tanto, una de las acciones favorecedoras de interacción padres-hijo se basa en permitir un acompañante elegido por la mujer durante el proceso de nacimiento ${ }^{3,4}$.

Si bien la literatura científica y las recomendaciones sobre el acompañamiento durante el parto y nacimiento estipuladas por la Organización Mundial de la Salud evidencian bienestar y satisfacción materna5, en las instituciones colombianas la implementación del acompañamiento a las parturientas es aún deficiente y no tiene soporte sólido en teorías de enfermería ${ }^{6}$. A medida que avanza el mundo, es urgente transmitir la voz de los nuevos padres en sus propias vivencias, quienes buscan involucrarse en el nacimiento de su hijo?.

Frente a estos hallazgos no se dudó en justificar una investigación para abordar el fenómeno con el objetivo de comprender la experiencia de los padres que vivenciaron el nacimiento de sus hijos en una institución de salud en Bogotá, Colombia, para lo cual se usó el método cualitativo con enfoque fenomenológico interpretativo y la investigación fue integrada con la Teoría del desarrollo humano de Rosemarie Rizzo Parse.

Según la evaluación critica de Fawcett, esta teoría cuenta con los criterios apropiados para ser aplicada en la práctica ${ }^{8}$. Recientemente, Parse invitó a realizar estudios apropiados, claros e informar con precisión los hallazgos ${ }^{9}$, en este sentido, la investigación soportada en teorías se integra cuando usamos un lenguaje más claro para describir la práctica ${ }^{10}$, como se evidencia en este estudio. 
Parse se ha centrado en las experiencias vividas por los seres humanos, su libertad y participación en la vida, vistos como seres indivisibles, imprevisibles y en constante cambio que, naturalmente, participan con el universo; es decir, siempre están vinculados con las ideas, el lenguaje, las tradiciones y otras personas ${ }^{11}$.

La teoría del desarrollo humano está constituida por los siguientes tres principios ${ }^{11}$

- Estructurar el significado: Se refiere a la importancia que una persona le asigna a algo que está relacionado con los momentos de la vida diaria y de su objetivo de vida. En este caso alude a cómo el padre vivencia la experiencia de estar presente en el nacimiento de su hijo a través de sus cinco sentidos.

- Configurar las pautas rítmicas: Parse menciona que las personas crean pautas de relación con los otros, las ideas, los objetos y las situaciones; mismas que dependen de sus prioridades, por lo que cambian constantemente cuando la persona integra nuevas experiencias. El estudio explicó cómo los padres se conectan con su recién nacido y la madre a través de una interacción mutua.

- Co-trascendencia con lo posible: Se refiere al cambio continuo y desarrollo de las personas a medida que eligen entre todas las opciones que aparecen en la vida diaria, sobre cómo ser, qué actitud tomar o con quién relacionarse. Aquí el hombre se ha transformado en un padre real con el nacimiento del hijo.

Parse afirma que el objetivo de la investigación con su teoría es descubrir la esencia de los fenómenos vividos para conseguir una comprensión más profunda de las experiencias humanas, y constituye un nuevo conocimiento sobre el desarrollo humano. Así, con fundamento en esta teoría se propuso comprender la experiencia de padres que vivenciaron el nacimiento de su hijo en una institución de salud.

\section{METODOLOGÍA}

Estudio cualitativo con enfoque fenomenológico interpretativo que se desarrolló entre julio de 2018 y marzo de 2019 en una clínica privada de Bogotá, Colombia. Este tipo de estudios permiten interpretar la forma en que el ser humano experimenta aquellos fenómenos de interés para enfermería, y así develar el cuidado desde el ser en sín $^{12}$.

La población estudiada fueron hombres que presenciaron el nacimiento de su hijo durante el parto institucionalizado, la muestra fue determinada por saturación teórica y quedó constituida por ocho participantes. Los criterios de inclusión fueron: padres mayores de 18 años que presenciaron el nacimiento de su hijo y que éste se hubiera resuelto por vía vaginal. Los criterios de exclusión fueron: complicación de salud en la madre o en el neonato y padres con alteraciones cognitivas.

El proceso para la recolección de la información fue de la siguiente manera: en el momento que una mujer gestante se encontró en trabajo de parto avanzado, la enfermera de la institución de salud se acercó a la sala de espera del área maternidad en busca del acompañante y lo invitó a participar en el parto. Cuando el compañero de la paciente aceptó, la enfermera le dio las indicaciones para el ingreso, tales como lavado de manos y uso correcto de un uniforme quirúrgico.

Desde el momento en que el padre se disponía a entrar al parto, la investigadora realizó observación participante (registrando en el diario de campo) y no participante hasta su regreso nuevamente a la sala de espera al finalizar el nacimiento de su hijo. Inmediatamente, la investigadora se acercó al padre en la sala de espera, se presentó e identificó, luego le presentó la investigación y explicó el estudio; cuando el padre aceptó y una vez firmado el consentimiento, se trasladaron a una sala de 
espera aislada, donde se realizó la entrevista semiestructurada con una duración promedio de 30 minutos, la cual fue grabada en su totalidad y transcrita por la misma investigadora.

Para la entrevista se tomó como pregunta orientadora: ¿Cómo fue la experiencia en el nacimiento de su hijo? Si bien la información se devolvió a los participantes con una llamada telefónica, el proceso fue válido y riguroso porque también fue grabada, transcrita en su totalidad, y tomada en cuenta nuevamente para el análisis. De igual forma, la primera entrevista también fue auditada por la asesora.

Los datos obtenidos fueron analizados mediante el método de Colaizzi (1978) siguiendo siete pasos: lectura de cada transcripción repetidamente, extracción de declaraciones significativas, formulación de significados, clasificación en categorías, clasificación en temas, integración de resultados en una descripción del fenómeno, y validación de hallazgos con los participantes ${ }^{13}$. La información fue organizada en una base de datos Excel con 251 declaraciones, 14 categorías y 3 temas emergentes. Posteriormente se analizó a la luz de la teoría de Parse.

En pro de la confiabilidad y validez ${ }^{14}$ fueron trabajados los criterios de rigor metodológico, en este caso la credibilidad se refiere a la descripción veraz de la experiencia ${ }^{15}$. Por consiguiente, se realizó observación participante y no participante, en la cual las expresiones de los participantes fueron tomadas literalmente, después la segunda investigadora revisó los resultados y fueron devueltos a los participantes, quienes estuvieron de acuerdo.

La transferibilidad es la posibilidad de trasladar los resultados de la investigación a otros contextos, por lo cual en este estudio se describieron detalladamente las condiciones contextuales, la información sociodemográfica de los participantes, se logró saturación teórica de la información y las entrevistas fueron grabadas y transcritas cuidadosamente.

A su vez, la replicabilidad hace referencia a la estabilidad de la información, para lo cual se describió gráficamente el proceso de recolección y análisis de datos, las transcripciones y categorización de información fueron analizados por la tutora.

La reflexibilidad busca asegurar la veracidad de la información, permite reconocer alcances y limitaciones del investigador, esto último se logró cuando la investigadora plasmó sus introspecciones, llevó un diario de campo y tuvo presente su papel como instrumento de investigación.

Según las pautas éticas recomendadas por $\mathrm{CIOMS}^{16}$, se obtuvo el consentimiento informado de los padres participantes de forma verbal y escrita, también se usaron seudónimos para proteger la identidad de los participantes. La investigación fue avalada por los comités de ética de la clínica y la Universidad Nacional de Colombia.

\section{RESULTADOS Y DISCUSIÓN}

Contexto: La institución de salud donde se llevó a cabo el estudio tiene una trayectoria de 71 años, es privada y se interesa en la atención humanizada. Durante el periodo de estudio tuvo en promedio 527 nacimientos por mes con un porcentaje de partos vaginales de $62 \%$ y cesáreas del $38 \%{ }^{17}$. Cuenta con 2 salas de parto en donde la lámpara de adaptación neonatal se encuentra dentro de la misma sala para evitar la separación madre e hijo, lo que permite que confluyan como un todo en un cuidado más humanizado. Ahí se permitía el ingreso del acompañante elegido por la madre cuando era solicitado y con la condición de que el parto fuera vaginal y en ausencia de complicaciones.

No había exigencia alguna hacia el padre que deseó ingresar, sólo les facilitaron un uniforme, gorro, tapabocas y cubre zapatos. Su ingreso fue únicamente al nacimiento, no al trabajo de parto; el tiempo de acompañamiento promedio fue de 30 minutos. Los padres participantes fueron todos 
del género masculino cuya edad estaba entre 21 y 46 años, con promedio de 30 años; $50 \%$ estaban casados; la residencia del 50\% estaba ubicada en estrato socioeconómico II (medio); el mayor nivel de escolaridad fue el profesional con 38\%; 100\% estaban empleados; por último, para 62.5\% era su primer hijo. Cabe resaltar que uno de los padres ya había presenciado el nacimiento de su primogénito.

En cuanto a los recién nacidos, 100 por ciento nacieron por parto vaginal y a término, es decir, por encima de las 37 semanas de gestación, con un peso entre 2405 y 3595 gramos, 3008 gramos en promedio; sin embargo, uno de ellos tuvo bajo peso al nacer BPN (peso menor de $2500 \mathrm{~g}$ ) y requirió Programa Madre Canguro sin necesidad de unidad neonatal. La talla varió entre 45 y $51 \mathrm{~cm}$, con un promedio de $49 \mathrm{~cm}$; respecto al género, 50\% fue masculino; cinco de los nacimientos ocurrieron en las horas de la mañana, dos en la tarde y uno en la noche.

Con relación a los antecedentes maternos de estos recién nacidos, todas las madres fueron de nacionalidad colombiana, la edad varió entre 20 a 34 años con un promedio de 27 años, y para 50\% de ellas era su segundo hijo.

Descripción del fenómeno: Los resultados de la investigación fueron representados en un árbol, como se muestra en la figura 1. En la raíz se evidencia la Teoría del Desarrollo propuesta por Rosemarie Rizzo Parse, esta teoría de enfermería se constituyó en el soporte para analizar la experiencia de los padres que vivenciaron el nacimiento de su hijo en una institución de salud.

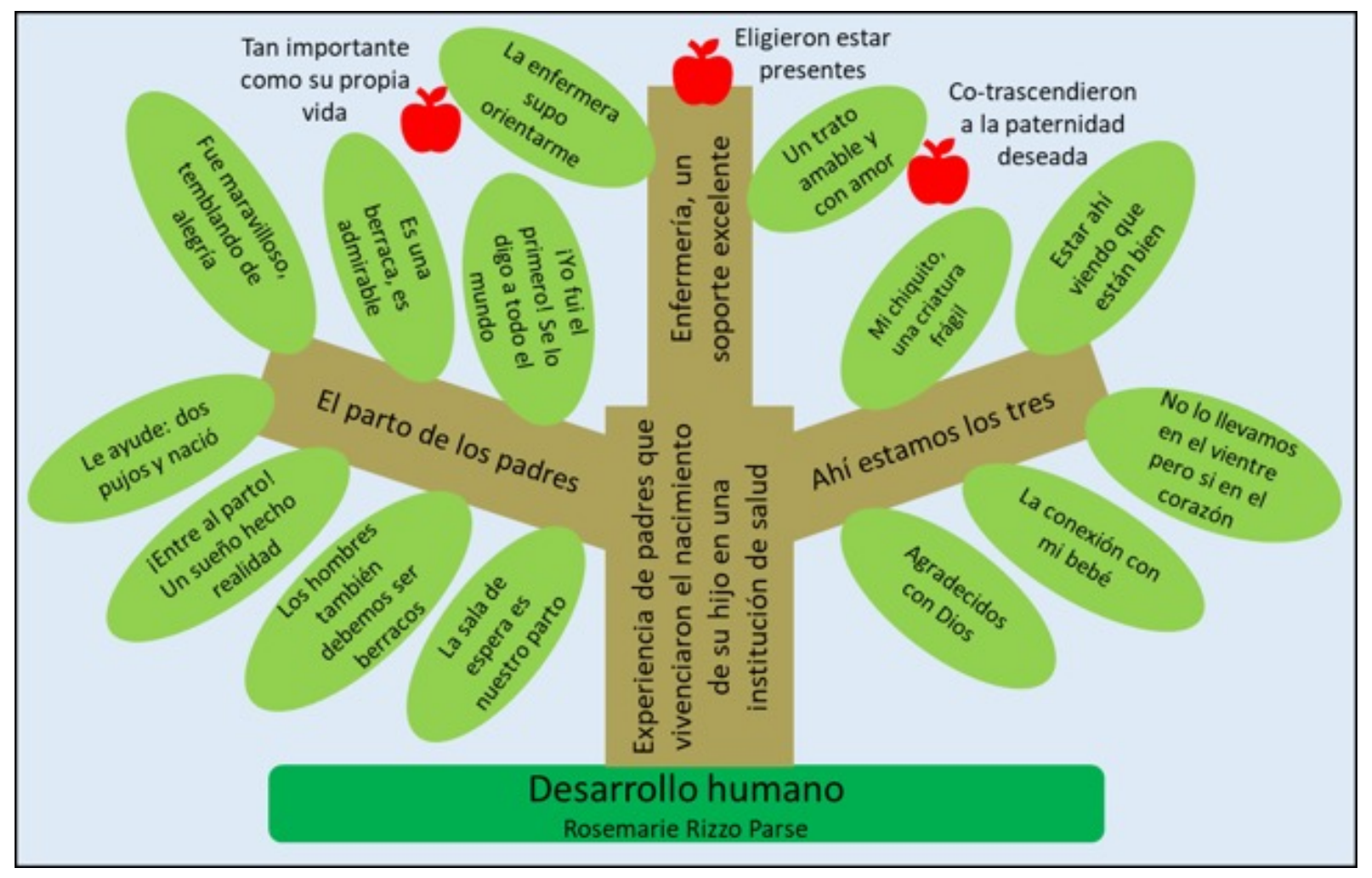

Figura 1. Temas emergentes de la investigación Experiencia de padres que vivenciaron el nacimiento de su hijo en una institución de salud

Por su parte, la presente investigación fue articulada con los tres principios de la teoría mencionada (estructurar el significado, configurar las pautas rítmicas, co-trascendencia con lo posible) 
representada en tres frutos del árbol denominados: Tan importante como su propia vida, eligieron estar presentes, y co-trascendieron a la paternidad deseada. De este modo, el tallo del árbol es el eje de la investigación Experiencia de padres que vivenciaron el nacimiento de su hijo en una institución de salud, en sus ramas y hojas se encuentran los tres temas obtenidos de esta investigación con sus respectivas categorías: El parto de los padres, ahi estamos los tres y enfermería, un soporte excelente.

\section{Tema 1: El parto de los padres}

Reúne la experiencia de los padres desde la eterna espera afuera de la sala de partos, la determinación y el deseo de entrar al parto, la posterior satisfacción por el aporte tras brindar su apoyo a la mujer y la admiración hacia ella.

- La sala de espera es nuestro parto. Los padres describieron sensaciones negativas durante el distanciamiento de su pareja debido a la incertidumbre, sintieron una barrera para el adecuado desarrollo del trabajo de parto. Un testimonio fue:

Esa ansiedad de bueno, ¿cómo por qué no me llaman?, ¿qué pasa?, ¿cómo estará la mamá?, si está sufriendo, si no está sufriendo, le da a uno dolor de estómago, como que se siente uno incómodo. Leví.

Lo anterior podría fundamentarse en el estrés que se genera en los padres a causa de factores como el temor ${ }^{18}$. Además, Etheridge et al..$^{19}$ encontraron que cuando los padres no recibieron información se sintieron excluidos, mientras que varios estudios ${ }^{20-22}$ evidenciaron que hacer a los padres partícipes es esencial para una experiencia positiva del parto y su bienestar mental. Por otra parte, aunque los padres del presente estudio anhelaban acompañar a sus parejas durante el trabajo de parto, estudios ${ }^{19,20}$ señalaron como esto supone enfrentarse a la impotente situación de ver a sus parejas adoloridas, además de enfrentarse a la crisis que genera las complicaciones inesperadas en salud de la madre o su hijo.

- Los hombres también debemos ser berracosa. Los padres asumieron el nacimiento del bebé como un proceso natural del ser humano y contrario a la creencia que los hombres se desmayan, ellos se sintieron valientes y aprovecharon la oportunidad para demostrarle a su pareja apoyo incondicional, como lo denotan los siguientes testimonios:

Si la mujer es tan berraca de tener un hijo y poner un hijo, porque eso es de berracas, el hombre también se debe parar de berraco y darle ánimo a su esposa y poder mirar a su hijo nacer; Joab. Yo le decía: yo estaba ahí para que se dé cuenta que siempre voy a estar al lado de ustedes... Que no tengo miedo de enfrentar alguna situación que se nos presente; Jared. Asimismo, Muñoz et al. ${ }^{23}$ mostraron a los padres motivados por involucrarse en el trabajo de parto y parto de sus parejas, brindándoles apoyo.

- ¡Entré al parto! Un sueño hecho realidad. Los padres deseaban estar en el nacimiento de sus hijos y agradecieron a quienes lo hicieron posible. Se relató lo siguiente:

La experiencia que siempre quise tener, gracias a Dios y la clinica... por habernos hecho cumplir este sueño tan maravilloso que es ver el nacimiento de nuestro segundo bebé; Leví. Según Howarth et al. ${ }^{20}$, los padres tuvieron la necesidad de participar en el nacimiento de sus hijos y esta participación se ha venido notando de manera natural4,24.

\footnotetext{
a Berraco: En el Nuevo diccionario de colombianismos del Instituto Caro y Cuervo se registra la palabra Berraco para referirse a una 'persona que por su talento o destreza sobresale en alguna actividad u oficio, o que se destaca por su fuerza física, audacia o valentía (Nota de la editora).
} 
- Le ayudé: dos pujos y nació. Los padres conocían la complejidad inherente al parto, por lo que buscaron la forma de ayudar a sus parejas. Lo hicieron acompañándolas y apoyándolas por medio del habla y el tacto, lo que aportó tranquilidad y fortaleza. Se sintieron satisfechos por incidir positivamente en el resultado. Un participante comentó: Sentí esa alegría de la mamá cuando me vio, sentirme ahí al lado... Creo yo que le ayudé hartísimo a la hora del nacimiento porque la vi como que estaba desesperada y en el momento que me vio abrió los ojos. Hola amor, vamos con esto y sentí la verdad que le había ayudado... La tranquilidad que sintió cuando yo la cogí de la mano; Leví. Respecto a este tema, Xue et al. ${ }^{25}$ encontraron resultados similares, recalcando los aportes de los padres en el bienestar materno infantil con su participación.

- iFue maravilloso! Temblando de alegría. La experiencia vivida por los padres al presenciar el nacimiento de sus hijos la describen como: bonita, maravillosa, incomparable, única y difícil de imaginar. Sin embargo, sus sensaciones fueron ambivalentes desde ansiedad, nerviosismo e intriga hasta alegría y satisfacción. Definitivamente el momento que llenó de emoción a los padres fue el ver nacer a su bebé como lo describe el siguiente relato:

Cuando lo vi salir no... iEse chiquitico! Fueron muchas emociones, o sea, imaginarme el momento que acabo de vivir es único... Verlo recién salido, con toda esa telita blanca, con sangre, llorando, con ganas de abrazarlo, sentirlo; Onán. Estos resultados se apoyan en un estudio ${ }^{20}$ donde los padres tuvieron alto grado de satisfacción con la experiencia y lo consideraron como un evento que impactó sus vidas.

- Es una berraca, es admirable. Los padres valoraron la fortaleza de sus parejas durante el nacimiento de sus hijos, se refirieron hacia ellas con admiración y orgullo por su tenacidad, en consonancia con el estudio ${ }^{20}$ que describe la satisfacción de los padres cuando sus parejas mantuvieron control sobre el proceso.

Yo he vivido varios días difíciles en mi carrera, pero un día dar a luz, traer una bendición es... Es de berracos... iEs una berraca, es admirable!... Es la que es y no me equivoqué; Onán.

- ¡Yo fui el primero! Se lo digo a todo el mundo. Para los padres haber sido la primera persona que ve, alza, pone el pañal y viste a su bebé los hizo sentir satisfechos y orgullosos, pues asignaron un significado especial al sentirse realmente padres.

Ya cuando me lo pasó, ahí fue cuando sentí la alegría, cuando sí sentí que fui papá... Que es mi hijo y que lo tengo en los brazos... El primero que lo ve de todas las dos familias; Abiel. De la misma forma, Howarth et al..$^{20}$ reportaron que los padres expresaron satisfacción por ser parte activa de los primeros instantes de vida de su hijo(a), por ejemplo, al cortar el cordón umbilical.

\section{Tema 2: Ahí estamos los tres}

El segundo tema describe las percepciones de los padres durante el primer encuentro con sus hijos/ as, la importancia de certificar el bienestar de sus parejas y bebés, el consecuente fortalecimiento de la paternidad y el agradecimiento a Dios por los beneficios recibidos.

- Mi chiquitico(a), una criatura frágil. Los padres en este estudio describieron a sus bebés como frágiles e indefensos debido a su pequeño tamaño y a que recién nacieron, esto, sumado a la poca experiencia, generó en ellos inseguridad tendiendo a ocultar su fuerza masculina para lograr ser delicados. Así lo relata un participante: 
Nervios de cogerla porque toda chiquita... La ropa le quedaba grande... Pero no, hermosa... Toda delicada, pero ahí me di mañitas y la pude cambiar; Hazael. Al comparar estos resultados con los encontrados por Castiblanco et al. ${ }^{26}$, se puede notar que los padres perciben a sus recién nacidos como frágiles, pequeños e indefensos, a pesar de que son bebés nacidos a término y con peso adecuado para la edad gestacional.

- Estar ahí viendo que están bien. Para los padres fue realmente importante haber participado del nacimiento de sus hijos(as) ya que fueron testigo del primer momento de sus vidas, motivo de orgullo para un padre. Ellos estuvieron atentos y dispuestos a admirar cada acontecimiento como lo refiere el siguiente testimonio:

El haber tenido la oportunidad de estar ahí cuando ella nació, de ver pues todo lo que hacen cuando ella nace, como la limpian, como le cortan, como le explican a uno, pues eso es, no sé, incomparable... Tuve el privilegio de verla desde que vio la luz entonces se lo contaré a ella cuando lo entienda... O sea, eso es una memoria que va a quedar ahí, imborrable; Lot.

En consonancia con lo anterior, Gouvêa de Matos et al.4 aseguraron que los padres sintieron alivio y alegría cuando verificaron el bienestar de su pareja e hijo, pues sintieron miedo por el sufrimiento y hasta por el riesgo de muerte que pudiera tener la diada.

- No lo llevábamos en el vientre, pero sí en el corazón. El deseo de ser buen padre llevó a los participantes a vincularse en el nacimiento de sus hijos(as), pues se convirtió en parte fundamental para el camino hacia la paternidad. Además, la experiencia motivó a los padres a criar y apoyar a sus hijos, lo que es esencial para la conformación de una familia. Aquí unos testimonios:

Los papás tienen que ser presenciales y que mejor ocasión que en el primer aliento de vida de su hijo... No se imaginan lo que llena a un padre presenciar ese proceso, si bien nosotros no llevamos el bebé en el vientre durante nueve, las cuarenta semanas, pero sí lo llevamos en el corazón. Entonces es esa sensación, ese es el parto de nosotros, que se traduce en lágrimas, esa es mi experiencia en la presencia del parto; Joab.

Se siente como más la unión, como el esfuerzo de los dos, como los lazos de la familia... Muy bueno... Para la creación de la familia, los pilares, los valores, principios, que desde aqui comienza todo, la formación de mi hijo; Onán. Estudios ${ }^{4,20,21}$ reafirman que estar presente en el nacimiento fortaleció en los padres las relaciones de pareja y el vínculo inmediato con sus bebés, fomentando su participación en el cuidado posterior de sus hijos(as) ${ }^{27}$.

- La conexión con mi bebé. Los padres buscaron la forma de interactuar con sus recién nacidos para transmitirles seguridad, esto lo hicieron alzándolos y hablándoles, posteriormente encontraron respuesta de sus bebés como el cese del llanto, el agarre de un dedo, la apertura y cierre de ojos. Desearon no separarse de ellos, confirmando el fortalecimiento del vínculo padre-hijo(a). Un testimonio fue:

La respuesta siempre fue abrir y cerrar los ojos, o sea, miraba para otros lados, pero cuando yo le hablaba abría y cerraba los ojos como en respuesta de que estaba feliz de verme... Quisiera hablarme, sentía que me hablaba con los ojos 'papá, gracias por darme esta vida', así lo sentí; Leví. Las interacciones con sus recién nacidos promueven la unión ${ }^{4}$, según Muñoz et al. ${ }^{23}$, el nacimiento que se vive en familia con la participación del padre impactaría positivamente en la vinculación familiar saludable. 


\section{Tema 3: Enfermería, un soporte excelente}

Este tema reúne la percepción de los padres acerca de la atención de enfermería durante el proceso de nacimiento de sus hijos(as), además del cuidado brindado hacia ellos y sus madres.

- La enfermera supo orientarme. Los padres apreciaron la guía continúa brindada por la enfermera, la claridad acerca del procedimiento a seguir durante su ingreso a la sala de parto y la recepción oportuna de información. Agradecieron la forma en que la enfermera los vinculó y enseñó el cuidado de sus parejas y recién nacidos(as), además refirieron la importancia de recibir preparación adecuada antes de su ingreso. Se relató lo siguiente:

Muy buena atención... Muy excelente calidad, y las personas también lo mismo, muy agradables... Por la forma en que me trataron a mí, me llegaron a preguntar que, si quería entrar al parto, yo sin saber, y me atendieron muy bien a mí... Me dijeron qué hacer... Me supieron orientar; Hazael. Estos hallazgos son similares a los de Howarth et al..$^{20}$, Xue et al. ${ }^{28}$ y Werner et $\mathrm{al.}^{22}$, cuyas investigaciones mostraron que los padres fueron orientados, apoyados, escuchados e incluidos, por lo que tuvieron una experiencia de parto más positiva, además de que mejoraron su participación ${ }^{23,25}$.

- Un trato amable y con amor. Los padres describieron el cuidado de la enfermera hacia sus esposas e hijo(as) como amable, compasivo, amoroso y paciente, generando en ellos tranquilidad y seguridad, tal como lo señalan otros estudios ${ }^{20,22}$ en donde resalta el profesionalismo y competencia primordial para los padres. Ellas siempre están al tanto y al cuidado, cómo consentían tanto a la esposita como al bebé es algo como persona y ser humano, me pareció muy excelente; Naín. Cabe destacar que fueron los padres quienes ilustraron el fenómeno de una forma original, amorosa y enriquecedora, constituyéndose en un aporte valioso para la disciplina de enfermería y para la sociedad.

\section{Análisis a la luz de la teoría del Desarrollo Humano de Parse}

En nuestro mundo actual se quieren resultados prácticos lo más rápido posible, gracias al uso de la teoría de Parse se desarrollaron y aplicaron conocimientos propios de la disciplina a la práctica de enfermería ${ }^{10}$. A continuación se evidencia la integración de la práctica en la investigación con dicha teoría.

Fue tan importante como su propia vida. Haciendo referencia al principio estructurar el significado, los padres asignaron un alto nivel de importancia a la experiencia de vivenciar el nacimiento de sus hijos(as) como un evento trascendental e irrepetible; su realidad estuvo constituida por su esposa, su nuevo hijo e hijos anteriores. Sus valores prioritarios fueron la familia, la empatía, la alegría, el amor y la responsabilidad por un nuevo ser que sienten como una extensión de su propio cuerpo y trae consigo el deseo de brindar protección, apoyo y crianza; estar presente desde el nacimiento, siendo este un momento de conexión entre la familia.

Ahora bien, durante este proceso la enfermera estuvo presente orientando a los padres. Parse anima a las enfermeras a respetar constantemente la forma y el tiempo que la persona requiera para asimilar el proceso de ilustración y comprensión de su situación, sin juzgarla.

Eligieron estar presentes. En cuanto al segundo principio configurar las pautas ritmicas, los padres tuvieron actitudes de apoyo y demostración de cariño hacia sus esposas durante el parto al tomarles la mano, acariciar su frente, besarlas, y susurrarles palabras de ánimo. Luego de vivir el nacimiento de sus hijos(as) expresaron admiración por la fortaleza con que ellas afrontaron el momento, agradecimiento por dar vida a sus hijos y nació el deseo de unirse más a ellas y fortalecer la confianza. 
Fue evidente un cambio en el patrón de comportamiento respecto al ingreso de un padre al parto, al decidir entrar a pesar del nerviosismo que les generó y dejando atrás los mitos, permitiendo descubrirse a sí mismos ante una nueva situación. Debido a los sentimientos ambivalentes que los padres experimentaron durante la preparación para ingresar a la sala de partos, éste se convierte en un momento propicio para que la enfermera entable un diálogo en donde ellos se sientan escuchados y estén más dispuestos a recibir orientación.

Co-trascendieron hacia la paternidad que deseaban. En el proceso de evolución de los padres, la elección de estar ahí los tres, con y para sus hijos, abrió las puertas a la posibilidad de conectarse con ellos desde su nacimiento, momento clave que facilitó la formación del vínculo padre e hijo(a) y el inicio de una relación más cercana, profunda y visible con sus recién nacidos. Al disponer de todos sus sentidos para el descubrimiento de nuevos sentimientos y sensaciones, los progenitores trascendieron hacia una paternidad activa y responsable que los lleva a cumplir su sueño de ser buenos padres, reafirmando que la familia es lo más preciado para ellos.

\section{CONCLUSIÓN}

La eterna espera afuera de la sala de parto de los padres es aliviada por la determinación y el deseo de entrar al parto con una posterior satisfacción. Los padres consideraron esencial su presencia en el nacimiento para apoyar y transmitir fortaleza a su pareja, donde ese primer encuentro con su recién nacido certifica el bienestar de la diada madre-hijo, fortaleciendo la paternidad traducida en Ahí estamos los tres. Finalmente, se comprendió que la experiencia de los padres que participaron en el nacimiento de su hijo/a fue una vivencia única y maravillosa, en la cual enfermería fue un gran soporte.

Los padres atravesaron el proceso de desarrollo humano empezando por la estructuración del significado fue tan importante como su propia vida, pasando por la configuración de sus pautas rítmicas que se refiere a que eligieron estar presentes y por último, co-trascendieron hacia la paternidad deseada.

Este estudio aporta a las enfermeras una guía para la atención y cuidado de los padres que experimentan el nacimiento de sus hijos/as, basada en una presencia real, ayudándolos a explorar sus sensaciones, prioridades, opciones, escucharlos y orientarlos, respetando la forma única y diferente de asimilar su proceso.

Los resultados denotan la importancia y trascendencia de la presencia de los padres en el nacimiento de sus hijos/as, por lo que se sugiere fortalecer la implementación de políticas, planes y guías de enfermería en este proceso. Aunado a esto, se concluye que el uso de la teoría de Parse fue el soporte teórico para comprender mejor la experiencia de los padres que vivenciaron el nacimiento de su hijo en una institución de salud en Bogotá.

Limitaciones: En el presente estudio se encontraron dos limitaciones; primera, los padres ingresaron a participar en el nacimiento de sus hijos al final del periodo expulsivo, por lo que el tiempo que dieron apoyo a su pareja fue limitado y dos, los padres confirmaron la veracidad de la información telefónicamente por asuntos laborales. Se sugiere en futuras investigaciones contemplar la posibilidad de realizar visitas domiciliarias para contar con una segunda entrevista presencial. 


\section{RESPONSABILIDADES ÉTICAS}

Protección de personas y animales. En esta investigación no se realizaron experimentos con personas o animales.

Confidencialidad. En esta investigación no aparecen datos personales que permitan identificar a los participantes, a quienes se les garantizó el anonimato y la confidencialidad de la información.

Conflicto de intereses. Los autores declaran no tener conflicto de intereses.

Financiamiento. Ninguno.

\section{REFERENCIAS}

1. Brüggemann OM, Ebele RR, Ebsen ES, Batista BD. No parto vaginal e na cesariana acompanhante não entra: discursos de enfermeiras e diretores técnicos. Rev. Gaúcha Enferm. 2015; 36(no.spe): 152-8. https://doi.org/10.1590/1983-1447.2015.esp.53019

2. Villarraga de Ramírez L. Atención del profesional de la salud al vínculo psico-afectivo madrepadre-hijo. av. enferm.1994; 12(2-3): 26-30. https://bit.ly/3cs4wne

3. Organización Mundial de la Salud. Recomendaciones de la OMS sobre el nacimiento. Declaración de Fortaleza,1985. Tecnología apropiada para el parto. Lancet. 1985; 2: 436-7.

https://bit.ly/31nOYuE

4. Gouvêa-de Matos M, Seixas-Magalhães A, Féres-Carneiro T, Nonato-Machado R. Construindo o vínculo pai-bebê: a experiência dos pais. Psico-USF. 2017; 22(2): 261-71.

http://dx.doi.org/10.1590/1413-82712017220206

5. Organización Mundial de la Salud. Recomendaciones de la OMS sobre intervenciones de promoción de salud para la salud materna y neonatal. Ginebra: OMS; 2015. https://bit.ly/39izyw8

6. Jiménez-Hernández GE, Peña-Jaramillo YM. Adherencia a las recomendaciones de la OMS en la atención del parto y nacimiento humanizado. Medellín, Colombia. Rev Univ Ind Santander Salud. 2018; 50(4): 320-7. https://doi.org/10.18273/revsal.v5on4-2018005

7. Tarouco-da Silva B, Santos-da Silva MR, Nunes-Bueno ME. Significant intra and extra-family events in the fatherhood construction process. Esc. Anna Nery. 2014; 18(4): 710-5.

https://doi.org/10.5935/1414-8145.20140101

8. Fawcett J. Criteria for evaluation of theory. Nurs Sci Q. 2005; 18(2): 131-5. https://doi.org/10.1177/089431840527482

9. Rizzo-Parse R. Manuscripts: what's missing? Nurs Sci O. 2020; 33(2): 109. https://doi.org/10.1177/0894318419898173

10. Robinson-Lane SG. Interdisciplinary nursing faculty and advancement of nursing knowledge. Nurs Outlook. 2021; SoO29-6554(21)00027-0. https://doi.org/10.1016/j.outlook.2021.02.003

11. Mitchel GJ, Bournes DA. Rosemarie Pizzo Parse. Desarrollo Humano. En: Raile-Alligood M, MarrinerTomey A. Modelos y teorías en enfermería. 7a ed. Barcelona: Elsevier. 2011.

12. Guerrero-Castañeda RF, de Oliva-Menezes TM, Lenise-do Prado M. La fenomenología en investigación de enfermería: reflexión en la hermenéutica de Heidegger. Esc. Anna Nery. 2019; 23(4): 1-7. https://doi.org/10.1590/2177-9465-ean-2019-0059

13. Shosha GA. Employment of Colaizzi's strategy in descriptive phenomenology: a reflection of a researcher. Eur Sci J. 2012; 8(27): 31-43. https://bit.ly/3DrgE6G

14. Cypress BS. Rigor or reliability and validity in qualitative research: perspectives, strategies, reconceptualization, and recommendations. Dimens Crit Care Nurs. 2017; 36(4): 253-63.

https://doi.org/10.1097/DCC.0000000000000253 
15. Noreña-Peña A, Alcaraz-Moreno N, Rojas JG, Rebolledo-Malpica DM. Aplicabilidad de los criterios de rigor y éticos en la investigación cualitativa. 2012; 12(3): 263-74. https://bit.ly/3w38Lh3

16. Consejo de Organizaciones Internacionales de las Ciencias Médicas (CIOMS)/Organización Mundial de la Salud. Pautas éticas internacionales para la investigación biomédica en seres humanos. Ginebra: CIOMS/OMS; 2002. https://bit.ly/3ssitay

17. Ministerio de Salud y Protección Social. Registro Único de Afiliados-RUAF. Aplicación Nacimientos y Defunciones. Bogotá, Colombia: MinSalud; actualización 2021. https://bit.ly/2P43chQ

18. Philpott LF, Leahy-Warren P, FitzGerald S, Savage E. Stress in fathers in the perinatal period: a systematic review. Midwifery. 2017; 55: 113-27. https://doi.org/10.1016/j.midw.2017.09.016

19. Etheridge J, Slade P. "Nothing's actually happened to me.": the experiences of fathers who found childbirth traumatic. BMC Pregnancy Childbirth. 2017; 17(1): 80.

https://doi.org/10.1186/s12884-017-1259-y

20. Howarth AM, Scott KM, Swain NR. First-time fathers' perception of their childbirth experiences. J Health Psychol. 2019; 24(7): 929-40. https://doi.org/10.1177/1359105316687628

21. Poh HL, Koh SSL, He HG. An integrative review of fathers' experiences during pregnancy and childbirth. Int Nurs Rev. 2014; 61(4): 543-54. https://doi.org/10.1111/inr.12137

22. Werner-Bierwisch T, Pinkert C, Niessen K, Metzing S, Hellmers C. Mothers' and fathers' sense of security in the context of pregnancy, childbirth and the postnatal period: an integrative literature review. BMC Pregnancy Childbirth. 2018; 18(1): 473. https://doi.org/10.1186/s12884-018-2096-3

23. Muñoz-Serrano M,Uribe-Torres $C$, Hoga L. Padre preparado y comprometido en su rol de acompañante durante el proceso de parto. Aquichan. 2018; 18(4): 415-25. https://doi.org/10.5294/aqui.2018.18.4.4

24. Johansson M, Fenwick J, Premberg A. A meta-synthesis of fathers' experiences of their partner's labour and the birth of their baby. Midwifery. 2015; 31(1): 9-18. https://doi.org/10.1016/j.midw.2014.05.005

25. Xue WL, He HG, Chua YJ, Wang W, Shorey S. Factors influencing first-time fathers' involvement in their wives' pregnancy and childbirth: a correlational study. Midwifery. 2018; 62: 20-8.

https://doi.org/10.1016/j.midw.2018.03.002

26. Castiblanco-López N, Muñoz-de Rodríguez L. Visión de las madres en el cuidado del hijo prematuro en el hogar. av. enferm. 2011; 29(1): 120-9. https://bit.ly/31lZFO6

27. Shorey S, Ang L, Goh ECL, Gandhi M. Factors influencing paternal involvement during infancy: a prospective longitudinal study. J Adv Nurs. 2019; 75(2): 357-67. https://doi.org/10.1111/jan.13848

28. Xue WL, Shorey S, Wang W, He HG. Fathers' involvement during pregnancy and childbirth: an integrative literature review. Midwifery. 2018; 62:135-45. https://doi.org/10.1016/j.midw.2018.04.013 